Communications in Physics, Vol. 20, No. 3 (2010), pp. 211-218

\title{
EFFECTS OF ELECTROPORATION ON BIOLOGICAL MEMBRANES EXPOSED TO HIGH POTENTIALS
}

\author{
NGUYEN THI THUY NHUNG, LE THANH TUNG, NGUYEN DUC GIANG, \\ NGO VAN THANH, AND NGUYEN AI VIET \\ Institute of Physics, VAST
}

\begin{abstract}
This study first considers that voltages of cellular organelle membranes could significanly surpass these of plasma membranes under the influence of ultrashort and high-intensity electric pulse. This is due to the voltages induced on the membrane. Using an approximate theory coordinated with the Kotnik's analytical method, considering the electroporation, we focus on the reactions of cell membranes placed in a trapezoidal pulse. Then, we discuss conductive power dissipations of normal cell and cancer cell generated by a sinusoidal exposure which include dielectric relaxation effects. In comparison with the complex numerical calculations of Joshi et al, our results are in very good agreement.
\end{abstract}

\section{INTRODUCTION}

Electroporation in biological cell is related with rapid structural rearrangement and formation of pores in the lipid layer, in respond to an externally applied electric field. It is leading to an important technique for inserting active molecules into cells and investigating electrically characteristic differences between normal and cancer cell. This article deals with the problem with a spherical cell with uniform interior, exposed to electric fields. The simplest model of a cell with an organelle is then obtained by incorporating into a cell another spherical body surrounded by a concentric shell $[1,2]$.

Dealing with this system, Joshi et al (2004) use a total numerical approach [2]. They simulate the spherical computational region including the cell by discretizing it in $r$ and $\theta$ directions. They also calculate the potentials including the dynamic pore model to deduce the changes of the conductivity of inner and outer membranes during pulse periods. This phenomenon is called electroporation. The pores are controlled by the Smoluchowski equation to diffuse across the energy landscape.This equation is so complex that it can only be solved by numerical method.

In keeping with the same system, Kotnik et al (2006) use the analytical approach in which they write down the roots of the Poisson quation in spherical coordinates [1]. Then they use the continuous conditions of the electric potentials $\boldsymbol{\Psi}$ for five regions and for the normal component of the electric current densities, $\Lambda(\partial \Psi / \partial \mathbf{r})$, with $\Lambda$ is the complex conductivity of a region. After solving a set of first-order equations, they achieve the analyical expressions of the transmembrane voltages, $\Delta \Psi_{m}$, as the difference between the 
potentials $\boldsymbol{\Psi}$ at the outer and inner surface of this membranes:

$$
\Delta \Psi_{m}=f E a \cos \theta
$$

where $\mathrm{E}$ is the strength of the electric field, $\theta$ is the polar angle measured with respect to the direction of the field, and $\mathrm{f}$ is a function reflecting the electrical and geometrical properties of the cell [5]. For the sinusoidal electric fields, $\Lambda$ becomes $\sigma+j \omega \epsilon$, where $\omega$ is the angular frequency of the field, $\sigma$ is the electric conductivity and $\epsilon$ is the dielectric permittivity. For the pulses, they substitute $\Lambda$ by $\sigma+s \epsilon$, the strength of the electric field $E$ by the Laplace transformation of the pulse from the time domain. Eventually, they use an inverse Laplace transformations to achieve the transmembrane voltages in the time domain, $\Delta \Psi_{\text {cell }}(t)=L^{-1}\left[\Delta \Psi_{\text {cell }}(s)\right]$ and $\Delta \Psi_{\text {org }}(t)=L^{-1}\left[\Delta \Psi_{\text {org }}(s)\right]$. An other theory approaching electroporation angles is Krassowska's approximate theory (1999) about modelling electroporation in a single cell develop an approximated model of electroporation, the model that bases only on an ordinary differential equation (ODE) [3,4]. The pore density $N(t)$ is governed by the ODE:

$$
\frac{d N}{d t}=\alpha e^{\left(V_{m} / V_{e p}\right)^{2}}\left(1-\frac{N}{N_{o}} e^{-q\left(V_{m} / V_{e p}\right)^{2}}\right) .
$$

And the pore current density is $N \cdot i_{e p}$, with the current through single pore [4]:

$$
i_{e p}=\frac{\pi r_{m}^{2} \sigma \nu_{m} R T}{F h} \times \frac{e^{\nu_{m}-1}}{\left(\frac{w_{o} \exp \left(w_{o}-n \nu_{m}\right)-n \nu_{m}}{w_{o}-n \nu_{m}} e^{\nu_{m}}-\frac{w_{o} \exp \left(w_{o}+n \nu_{m}\right)+n \nu_{m}}{w_{o}+n \nu_{m}}\right)}
$$

where $V_{m}$ is the transmembrane voltage on the membrane and $\nu_{m}=V_{m} F / R T$ is the nondimentional transmembrane voltage. Although having a simpler model of electroporation, they also use the discretizing method in calculation potentials. Values of all paremeters are given in Table 1

\section{METHOD}

In this study, we improve the combined method in investigating the properties transmembrane voltages of cancer cells and normal cell as well as the differences between them in exposure to the triangular and trapezoidal pulses. We use the analytical equations of voltages from Kotnik et al and also include the dynamic conductivity properties by the approximated model of Krassowska et al. All the kinds of cells and pulses are taken from Joshi's paper and the results are compared to Joshi to judge the efficiency of the combined models. We use the continuum model of pore where the pore resistance, $R_{p}=h /\left(\pi s r^{2}\right)$, and the input resistance, $R_{i}=1 /(2 s r)$ are connected in series.

In addition, we expand the analytical calculation of Kotnik et al to solve a problem of power dissipation of both cells with dielectric relaxation processes. We list here all the factors of cells and pulses used in this article [2] (Table 2). 
Table 1. Geometric, electrical, and electroporation parameters [3].

\begin{tabular}{||l|c|l||}
\hline \hline Symbol & Value & Definition \\
\hline$\alpha$ & $100.0 \mathrm{~cm}^{-2} \mathrm{~ms}^{-1}$ & Electroporation parameter \\
\hline $\mathrm{q}$ & 2.46 & Electroporation constant \\
\hline$V_{e p}$ & $258 \mathrm{mV}$ & Characteristic voltage of electroporation \\
\hline$r_{m}$ & $0.76 \mathrm{~nm}$ & Pore radius \\
\hline$a$ & $50.0 \mathrm{~nm}$ & Cell radius \\
\hline$h$ & $5.0 \mathrm{~nm}$ & Membrane thickness \\
\hline$n$ & 0.15 & Relative entrance length of the pore \\
\hline$g_{l}$ & $0.19 \mathrm{mS} / \mathrm{cm}^{2}$ & Specific membrane resistance \\
\hline$E_{l}$ & $283.75 \mathrm{mV}$ & Reversal potential of ionic current \\
\hline$C_{m}$ & $0.95 \mathrm{mF} / \mathrm{cm}^{2}$ & Specific membrane capacitance \\
\hline$s_{i}$ & $4.55 \mathrm{mS} / \mathrm{cm}$ & Intracellular specific conductivity \\
\hline$s_{e}$ & $50.0 \mathrm{mS} / \mathrm{cm}$ & Extracellular specific conductivity \\
\hline$T$ & $295 \mathrm{~K}\left(22^{\circ} \mathrm{C}\right)$ & Temperature \\
\hline$R$ & 8.314472 & Universal gas constant \\
\hline$s$ & $13.0 \mathrm{mS} / \mathrm{cm}^{-2}$ & Conductivity of aqueous solution in pores \\
\hline$N_{o}$ & $1.5 \times 105 \mathrm{~cm}$ & Equilibrium pore density when $V_{m}=0 \mathrm{mV}$ \\
\hline$w_{o}$ & 2.65 & Energy barrier within pore \\
\hline \hline
\end{tabular}

\section{STEPS AND RESULTS}

\section{III.1. Trapezoidal Pulses}

Firstly, we use the Laplace-transform method in calculating the transmembrane voltages of a typical cell exposured to the two kinds of trapezoidal pulses as in the first column of Table. 2: a trapezoidal pulse with intensity $45 \times 10^{5} \mathrm{~V} / \mathrm{m}$, rise and fall time $10 \mathrm{~ns}$ and duration $70 \mathrm{~ns}$.

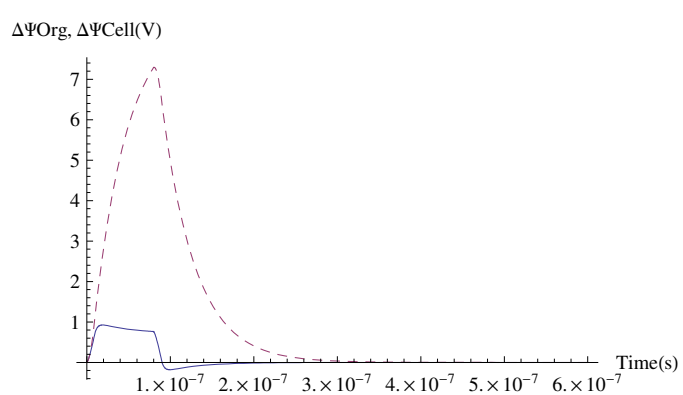

Fig. 1. The time courses of votages induced by a trapezoidal pulse of normal cell. Solid: cell, dashed: organelle

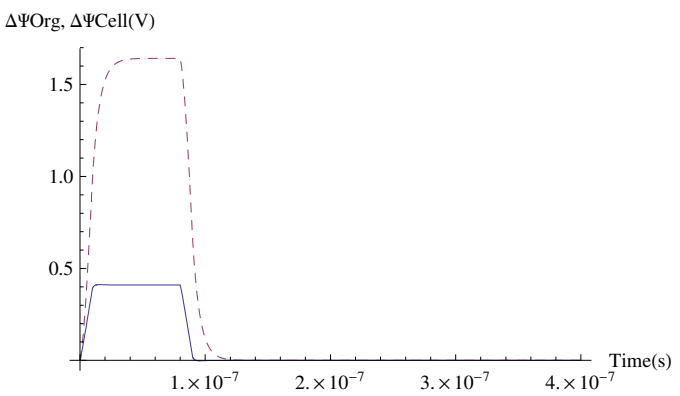

Fig. 2. The time courses of votages induced by a trapezoidal pulse of cancer cell. Solid: cell, dashed: organelle 
Table 2. Typical parameters of cells and electric pulses for this analysis [2].

\begin{tabular}{||l|c|c|c||}
\hline \hline & Typical cells & Nor B-cells & Malig B-cells \\
\hline Conductivities $(S / m)$ & & & \\
\hline Evironment & 0.6 & 0.6 & 0.6 \\
\hline Cell membrane & 0.0 & $5.6 \times 10^{-5}$ & $9.1 \times 10^{-6}$ \\
\hline Cytoplasm & 0.6 & 1.31 & 0.48 \\
\hline Nuclear envelope & 0.0 & $1.11 \times 10^{-2}$ & $4.4 \times 10^{-3}$ \\
\hline Nucleoplasm & 0.6 & 2.04 & 1.07 \\
\hline Relative permittivity & & & \\
\hline Evironment & 80 & 80 & 80 \\
\hline Cell membrane & 8 & 12.8 & 9.8 \\
\hline Cytoplasm & 80 & 60 & 60 \\
\hline Nuclear envelope & 4 & 106 & 60.3 \\
\hline Nucleoplasm & 80 & 120 & 120 \\
\hline Geomatry parameters $(\mu m)$ & & & \\
\hline Cell radius & 5 & 3.3 & 5.2 \\
\hline Outer mem thickness & 0.005 & 0.007 & 0.007 \\
\hline Nucleus radius & 1 & 2.8 & 4.4 \\
\hline Nucleus mem thickness & 0.01 & 0.04 & 0.04 \\
\hline \hline
\end{tabular}

Figures 1 and 2 depict that the voltages of inner membranes are higher than these of outer membranes in both cases. Comparing these values with Joshi's ones, however, we found that the values of potentials are larger than Joshi's calculations by a few volts. This is due to the fact that the analytical calculation of Kotnik does not consider the dynamic properties of the conductivity biological of membranes. Due to the electroporation phenomenon, under an external voltage of above $1.0 \mathrm{~V}$, the conducting pores appear on membranes, and therefore, increase the effective conductivity of membrane. This leads to a decrease in the induced voltages on the membranes.

These inadequate results can be made good by using an approximated model of current through pores (Alan Barnett), mentioned in the paper of Krassowska et al. [4]. We consider here the pore current described by the Eq. (3). The point is simple: we subtract the voltages induced by the appearances of the pores.

$$
\begin{aligned}
& \Delta \Psi_{\text {ce }}=\Delta \Psi_{\text {cell }}-\Delta V_{m, \text { cell }}, \\
& \Delta \Psi_{\text {or }}=\Delta \Psi_{\text {org }}-\Delta V_{m, \text { org }} .
\end{aligned}
$$

with $\Delta V_{m, \text { cell }}$ and $\Delta V_{m, \text { org }}$ is respectively the voltages induced on outer and inner membrane. This voltage can be considered to be the multiplication between the pore current and the resistance of a single pore. If we consider the pores to be parallel connected, the overall decrease of voltage can be the decrease of voltage on a single pore. Note that in the Eq. 3, $r_{m}$ is the radius of pores, in which the pore energy has a local minimum, $r_{m}=0.8 \mathrm{~nm}$, and has a largest probability to appear [3]. The voltages made by pores are 
calculated by the equations:

$$
\begin{aligned}
& \Delta V_{m, \text { cell }}=i_{\text {cell }, e p} \cdot\left(R_{\text {cell }, p}+R_{\text {cell }, i}\right) . \\
& \Delta V_{m, \text { org }}=i_{\text {org }, e p} \cdot\left(R_{\text {org }, p}+R_{\text {org }, i}\right) .
\end{aligned}
$$

with $i_{c e l l, e p}, i_{\text {org }, e p}$ are calculated by Eq.3 in which $V_{m}$ is respectively replaced by $\Delta \Psi_{\text {cell }}$ and $\Delta \Psi_{\text {org }}$ Using this update, we re-draw the membranes voltages for two pulses above. We see here the good agreement between numerical and analytical approaches.

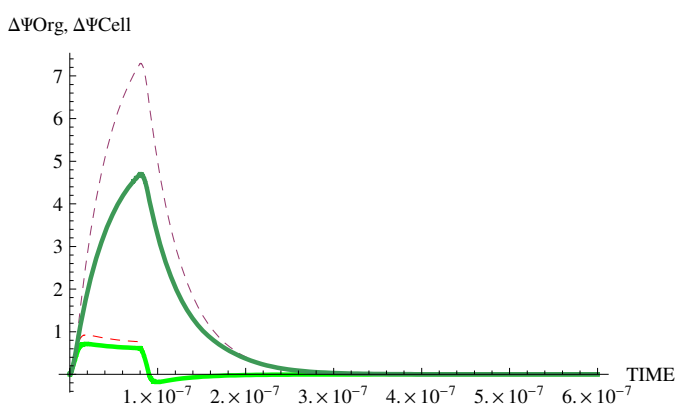

Fig. 3. The time courses of votages induced by a trapezoidal pulse of normal cell. Dashed: Kotnik' calculation [1] (red: outter, purple: inner), thick: our calculation.(green: outer, dark: inner) $V_{m}$.

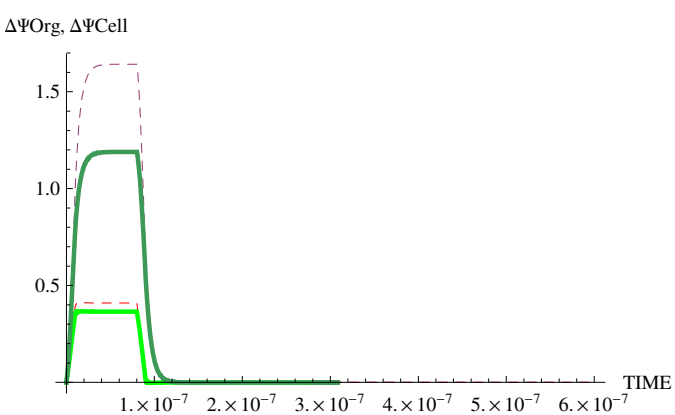

Fig. 4. The time courses of votages induced by a trapezoidal pulse of normal cell. Dashed: Kotnik' calculation [1] (red: outter, purple: inner), thick: our calculation.(green: outer, dark: inner).

Figures 3 and 4 point out that the voltages of inner membranes always surpass these of outer membranes of both normal and cancer cells when they are exposed to the electric pulse. Particularly, potentials of cancer membranes (for inner one: about $0.4 \mathrm{~V}$, for outer one: about $1.2 \mathrm{~V}$ ) are considerably less than potentials of normal membrane (for inner one: about $0.7 \mathrm{~V}$, for outer one: about $4.5 \mathrm{~V}$ ).

The effect of electroporation on outer and inner membrane is different. To clarify this point, we use the Eq. (2) to calculate the pore densities on these membranes versus time. We use a trapezoidal pulse with the strength of the electric field is $45 \times 10^{5} \mathrm{~V} / \mathrm{m}, 70$ ns of duration and $10 \mathrm{~ns}$ of risetime and falltime. The numerical intergrations are taken with the time-dependent membrane voltages calculated as before.

Obviously, Fig. 5 points out that the value of pore density of inner membrane is always higher than this of outer membrane. The peak of the inner pore density (about $10^{19}$ ) is proximately $15-16$ order larger than that of outer membrane. This point indicates that ultrashort pulse can excite the inner membrane and the electroporation appears, the pore current increases dramatically. For this case, the thermal effect can be ignored because of the short pulses and the low conductivity of biological membranes.

\section{III.2. Power Dissipation with Dielectric Relaxation}

We continue to apply our calculation to the normal and cancer cells, interacted with a triangular pulse with $10 \mathrm{~ns}$ rising edge and $50 \mathrm{~ns}$ falling edge and the magnitude is 45 $\mathrm{kV} / \mathrm{cm}[2]$. 


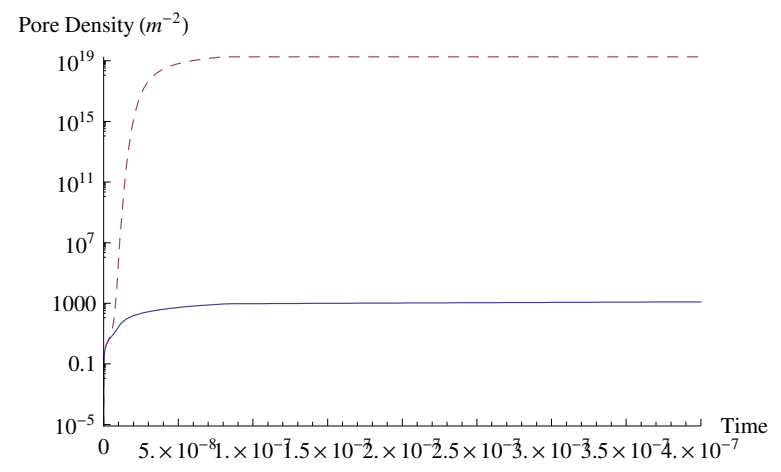

Fig. 5. Pore density versus time of a cancer B cell in short pulse case (solid: outer membrane, dashed: inner membrane)

Firstly, we use the parameters of membranes as in the second and the third columns of Table. 2. We plot here these two results of transmembrane voltages in two cases.

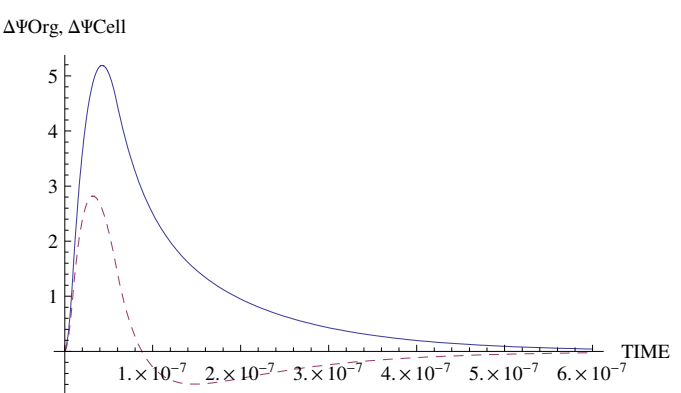

Fig. 6. Cell (continuous) and inner (dashed) voltages of normal B- cell, using typical parameters.

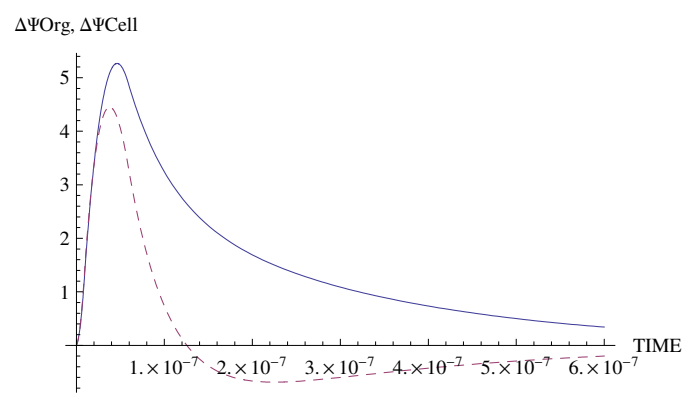

Fig. 7. Cell (continuous) and inner (dashed) voltages of cancer B- cell, using typical parameters.

We easily see that the voltages of outer membrane always higher than inner membrane in Figs. 6, 7. It is demonstrated that conductivities of transmembranes notably influence electroporation. With the triangular case, the typical parameters do not make electroporation take place.

According to the Eq. (2), after a period of time, the pore density reaches $N_{o}$ and therefore, the conductivity of membrane would be higher than in the initial case $N=0$. Therefore, we choose the effective conductivities of the outer and inner normal membranes according to Joshi's paper. That means $\sigma_{1 m}=1.1 \times 10^{-2}$ and $\sigma_{2 m}=3.1 \times 10^{-2}$ as the initial values. The latter one is chosen as in the very first time of the simulation, the outer membrane's conductivity changes dramatically to that value due to electroporation [2]. For the cancer cells, they are $5.2 \times 10^{-2}$ for the outer and $8.6 \times 10^{-2}$ for the inner membranes, respectively.

The only changes in the conductivity give a dramatic difference as in Figs. 8, 9. With this effective conductivity of outer and inner membranes, the results are in very 
good agreement with Joshi's results and shows the effects of ultrashort pulses in exciting sub-stuctures.

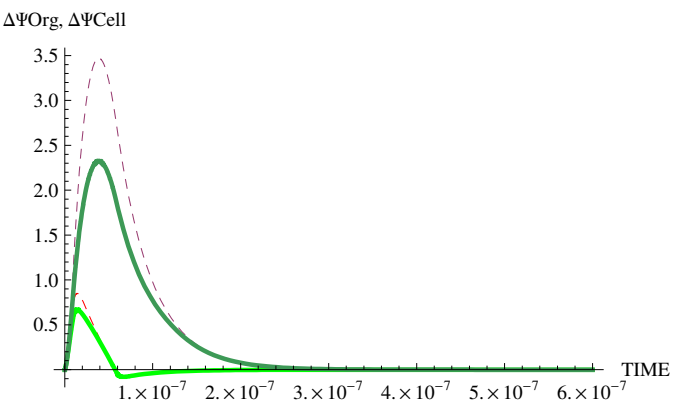

Fig. 8. The voltages of normal B- cell Dashed: Kotnik' calculation method (red: outter, purple: inner), thick: our calculation method (green: outer, dark: inner), using updated parameters.

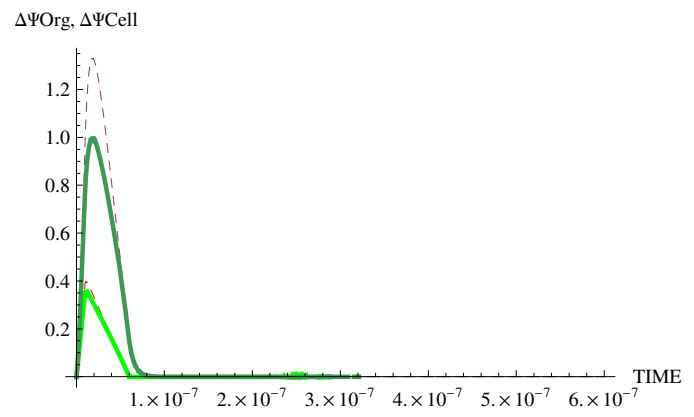

Fig. 9. The voltages of cancer B- cell Dashed: Kotnik' calculation method (red: outter, purple: inner), thick: our calculation method.(green: outer, dark: inner), using updated parameters.

In addition, it is well known that a variety of profound biochemical and biophysical effects can be led by exposure of biological cells to electric field. In common, evaluation of these effects is based on the power dissipation caused by the exposure. The power dissipation per init volume is given by $P=\sigma \bar{E}^{2}$.

Because of the analytical approach, we instantaneously have the distribution of the potential for the entire space and entire period of pulse time. Therefore, it is convenient to calculate the dissipation energy of the pulses giving to the cells. Although the power dissipation is assessed through the bulk properties of the tissue, we ignore all the nonuniformities of in the distribution of the field as well as the heat flow that will redistribute between regions. This is because the exposure takes place in very short exposure time, 10 ns [5]. To compare, we also apply the the infinitive sinusoidal fields towards normal and cancer cells, Figs. 10, 11. In this calculation, we also include the dielectric relaxation, an addition component of power dissipation resulted from rotation or flexion of molecular dipole, which dominants in the higher frequency field, $\left(10^{12} \mathrm{~Hz}\right)$.

Our calculations point out that the peak values of power dissipation of a cancer cell and normal cell are somewhat the same (about $10^{10} \mathrm{~W} / \mathrm{m}^{3}$ ). There is only a frequency range from $10^{6}$ toward $10^{8} \mathrm{~Hz}$ in which the energy dissipated in the inner membranes surpasses the external one, but similar for both cases. Excepting this interval, the values of $P$ of inner membrane of normal cell are higher than those of cancer membrane.

\section{CONCLUSION}

To conclude, we so far improve the model of cell membrane exposed to a $n s$ ultrashort, high-intensity pulse. Basing on the analytical Laplace- transform method of pulses, we include the effect of dynamic conductivity of cell membranes to achieve better results, which are in good agreement with numerical calculations. We also concern the differences 


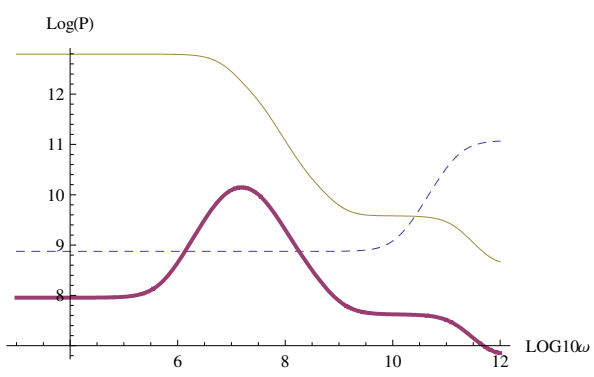

Fig. 10. Dissipation power of normal B- cell versus frequency, (thick: inner mem, solid: outer mem, dashed: external environment).

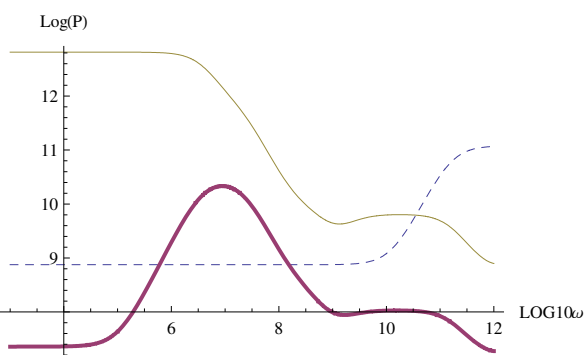

Fig. 11. Dissipation power of cancer B- cell versus frequency, (thick: inner mem, solid: outer mem, dashed: external environment).

of the responses between cancer and normal B-cells. In detail, the potetials of cancer cells are less than the potentials of normal cell for a few volts.

Some difficulties, however must be noticed to enhance our results: The time-dependent conductivity needs to be recreated based on the theory of Krassowska et al. The predicament is that we have to choose the initial pore density without a connection between the equilibrium density of pores and the effective conductivies of membranes.

\section{REFERENCES}

[1] Tadej Kotnik and Damijan Miklavcic, Biophys J. 90(2) (2006) 480-491.

[2] R. P. Joshi, Qin Hu, K. H. Schoenbach, Plasma Science, IEEE Transactions, 32 (4) (2004) 1677 1686.

[3] John C. Neu and Wanda Krassowska, Phys. Rev. E, 59 (1999) 3471 - 3482.

[4] Katherine A. DeBruin and Wanda Krassowska, Biophysical Journal 77 (3) (1999) 1213-1224.

[5] Tadej Kotnik and Damijan Miklavcic, Biophysical Journal 90 (2) (2006) 480-491.

[6] Lodish, Berk, Zipursky, Baltimore Molecular cell biology Fifth editor. Media Connected

[7] Jame C.Weaver, Yu.A.Chizmadzhev, Bioelectrochemistry and Bioenergetics Journal 41 (1996) 135160.

[8] I.G Abidor, V,B. Arakelyan, L.V Chemomordik et al. Bioelectrochemistry and Bioenergetics Journal 6 (1979) 37-52.

[9] R. Benz, F. Beckers and U.Zimmermann, J. Membr. Biol 48 (1979) 181 -204.

[10] A. J. Wagner, S. May, Eur Biophys J 36(4-5) (2007) 293-303

[11] O. I. Fisun, Bioelectromagnetics, 14(1) (1993) 57-66

[12] T. Ando, Rev. Mod. Phys. 54 (2) (1882) 437-672

[13] J. M. Pitarke, V. M. Silkin, E. V. Chulkov, P. M. Echenique, Rep. Prog. Phys. 70 (2007) 1-87

[14] F. Stern, Phys. Rev. Lett. 18 (1967) $546-548$

[15] A. Fetter, J. D. Walecka Quantum Theory of Many-particle Systems, McGraw-Hill Book Company

[16] K. J. Blinowska, W. Lech, A. Wittlin, Phys. Lett. A 109(3), (1985), 124-126

[17] M. A. Rojavin, M. C. Ziskin, QJM 91 (1998) 57-66.

[18] P. Debackere, S. Scheerlinck, P. Bienstman, R. Baets, "A Biosensor Based on Surface Plasmon Interference", LEOS Benelux Annual Symposium, Netherlands, (2006)

[19] A. G. Rojo, J. Physics: Condensed Matter. 11 (5) (1999) 31-52; E. Manousakis, Phys. Lett. A342 (5-6) (2005) 443-447

[20] H. Raether, "Surface Plasmons on Smooth and Rough Surfaces and on Gratings", Springer-Verlag, Berlin, Heidelberg, New York, London, Paris, Tokyo 